\title{
Effect of Teriparatide on pain relief, and quality of life in postmenopausal females with osteoporotic vertebral compression fractures, a retrospective cohort study
}

\author{
Zhipeng Chen ${ }^{1,2 \#}$, Wei Lin ${ }^{1,2 \#}$, Shengli Zhao ${ }^{1,2}$, Xiaoyi Mo ${ }^{1,2}$, Weiquan Yuan ${ }^{1,2}$, Wing Hoi Cheung ${ }^{3}$, \\ Dan Fu ${ }^{4}$, Bailing Chen ${ }^{1,2}$
}

${ }^{1}$ Department of Spine Surgery, The First Affiliated Hospital of Sun Yat-sen University, Guangzhou, China; ${ }^{2}$ Guangdong Provincial Key Laboratory of Orthopedics and Traumatology, The First Affiliated Hospital of Sun Yat-sen University, Guangzhou, China; ${ }^{3}$ Department of Orthopaedics and Traumatology, Prince of Wales Hospital, The Chinese University of Hong Kong, Hong Kong, China; ${ }^{4}$ Department of Orthopedic, Kiang Wu Hospital, Macau, China

Contributions: (I) Conception and design: B Chen, WH Cheung, D Fu; (II) Administrative support: B Chen; (III) Provision of study materials or patients: B Chen, Z Chen; (IV) Collection and assembly of data: Z Chen, W Lin, S Zhao, X Mo, W Yuan; (V) Data analysis and interpretation: Z Chen, W Lin; (VI) Manuscript writing: All authors; (VII) Final approval of manuscript: All authors.

\#These authors contributed equally to this work.

Correspondence to: Bailing Chen, MD, PhD. Department of Spine Surgery, Guangdong Provincial Key Laboratory of Orthopedics and Traumatology, The First Affiliated Hospital of Sun Yat-sen University, Guangzhou 510080, China. Email: chenbl96@mail.sysu.edu.cn.

Background: Osteoporotic vertebral compression fracture (OVCF) is a common disease in elderly population, which could cause serious back pain and has a substantial impact on patients' health-related quality of life (HRQoL). The aim of this study was to identify the effect of Teriparatide as a conservative treatment on reducing back pain, and improving quality of life for postmenopausal women with osteoporotic vertebral fractures.

Methods: In a 12-month, retrospective study, 112 postmenopausal women with OVCFs were assigned to Teriparatide group (20 $\mu \mathrm{g}$ Teriparatide, subcutaneous, once daily, $\mathrm{n}=38$ ) or control group (500 mg calcium and 400-800 IU Vitamin D per day, oral administration, n=74) according to patients' choices between January 2016 and October 2018. Patient-reported outcomes scores including the visual analogue score (VAS), Oswestry disability index (ODI), and short form 36 questionnaire (SF-36) were assessed at baseline, the $3^{\text {rd }}$ months, the $6^{\text {th }}$ months and 1 year after treatment.

Results: Treatments with Teriparatide or calcium plus vitamin D supplements had significant effect on improvement of patients' back pain as well as HRQoL, with significantly reduced VAS and ODI and increased SF-36 physical component summary (PCS) and mental component summary (MCS) scores. At the endpoint, Teriparatide showed better therapeutic effect, with greater reductions in VAS and ODI and more increases in SF-36 PCS and MCS scores. However, more adverse events (AEs) were found in Teriparatide group, but symptoms were relatively mild and of short duration.

Conclusions: In postmenopausal women with OVCFs, the consequent persistent back pain and impaired HRQoL, treatment with Teriparatide was associated with more profound therapeutic effects and more AEs compared with calcium plus vitamin D supplements.

Keywords: Teriparatide; postmenopausal osteoporosis; back pain; health-related quality of life (HRQoL); spinal fractures

Submitted Nov 24, 2020. Accepted for publication Jan 13, 2021.

doi: 10.21037/apm-20-2333

View this article at: http://dx.doi.org/10.21037/apm-20-2333 


\section{Introduction}

Osteoporosis, a worldwide age-related metabolic bone disease in elderly population, is characterized by low bone mass and microarchitectural deterioration of bone tissue (1). Osteoporotic vertebral compression fractures (OVCFs) are known as the most common type of fracture associated with skeletal fragility, affecting more than $30 \%$ of women aged 75 and even $50 \%$ of women aged 85 or older $(2,3)$. It is emerging as a major serious public health problem worldwide, especially in developing countries, and brings severe challenges to global health and social service sector because of aging of population.

Back pain, the first appeared and the most common clinical manifestation of fractures, causes significant disability, functional limitations and loss of independence. In addition to back pain, patients are increasingly becoming concern about the burden on health-related quality of life (HRQoL) (4,5). It is known that OVCFs can result in reduced physical functions and psychosocial impairments, which eventually make the patients more prone to depression, loss of productivity, independence, and social isolation $(2,6)$. All of these are the reasons of significant reduction of HRQoL (7-9). What's more, the annual hospitalization expenditures associated with vertebral fractures were estimated at 500 million dollars in the United States and about Euro 377 million in Europe, and majority of these hospital costs were related to the treatment of back pain and the resultant impaired HRQoL $(10,11)$. Consequently, it is vitally important to improve HRQoL and relieve back pain in patients with OVCFs (12).

Among many attempts to improve HRQoL and relieve back pain with pharmacological therapies in the patients with very advanced osteoporosis or OVCFs, Teriparatide has drawn more and more attention in the past two decades because of its central role in regulation of calcium and phosphate metabolism. Besides, Teriparatide is the first anabolic agent recommended for severe osteoporosis that offers the efficacy of increasing new bone formation and improving bone quality (13-15). Therefore, Teriparatide has been reported to reduce the risk of vertebral fracture $(14,16)$, and have the potential to accelerate fracture healing, form new bone, mitigate the progression of osteoporosis, heal and stabilize pre-existing fractures or microdamages in patients with advanced osteoporosis $(15,17)$. Yet, to date, sparse data exists regarding the efficacy of Teriparatide on relieving back pain and improving HRQoL of postmenopausal females with OVCFs.
This study aims to assess whether use of Teriparatide was associated with better therapeutic efficacy in postmenopausal women with OVCFs compared with calcium plus vitamin D supplements. The safety profiles also were assessed. We present the following article in accordance with the STROBE reporting checklist (available at http://dx.doi.org/10.21037/apm-20-2333).

\section{Methods}

This was a 12-month retrospective cohort study. The study was performed in compliance with the ethical principles outlined in the World Medical Association's Declaration of Helsinki (as revised in 2103) and was approved by the Institutional Review Board of the First Affiliated Hospital of Sun Yat-sen University (No. 2020-149) and individual consent for this retrospective analysis was waived. Patients were recruited from our osteoporotic-fracture clinic or our spinal surgery clinic in the First Affiliated Hospital of Sun Yat-sen University between January 2016 and October 2018. 112 ambulatory postmenopausal women aged 55 years or older with established OVCFs were enrolled (18). Prior use of any anti-resorptive drugs was not allowed to avoid the aftereffects of previous drugs. Subjects were excluded from the study if they had metabolic bone disease other than postmenopausal osteoporosis; imminent need for kyphoplasty or vertebroplasty; and evidence of significant pathology related to back pain which was difficult to be interpreted by osteoporotic vertebral fracture. Besides, subjects with uncontrolled high blood pressure, liver disease, secondary osteoporosis or abnormal thyroid function, renal and hepatic dysfunction, medical conditions such as cancer, severe sleep apnea, chronic obstructive pulmonary disease, severe lactose intolerance, and current use of tobacco were excluded.

Subjects recruited were assigned to two groups according to their own choices between Teriparatide and calcium plus vitamin D supplements. Subjects in the Teriparatide group received once-daily subcutaneous self-injection of Teriparatide $(20 \mu \mathrm{g} /$ day $)$, while subjects in the control group received daily supplements of $500 \mathrm{mg}$ calcium and 400 to 800 IU vitamin D.

DXA scans at lumbar spine and proximal femur were performed at baseline. Blood samples were collected and analyzed the serum phosphorus, calcium, and alkaline phosphatase at baseline as well. The initial assessments of patient-reported outcome scores were performed on site, and the following assessments were conducted at months 3 , 
6 , and 12 after initiation of treatment at each study visit or by phone call follow-up.

Demographic variables such as age, sex, body-mass index (BMI), and total vertebral fractures at baseline were recorded. Patient-reported outcomes scores, including the visual analogue score (VAS), Oswestry disability index (ODI), and short form 36 questionnaire (SF-36) were employed to evaluate each patient's symptoms and HRQoL, respectively. In brief, patients were asked to rate their back pain on an VAS, the most reliable and reproducible tool for the measurement of pain (19). It ranges from 0 to 10 , representing no back pain at all and the worst possible back pain, respectively. The disease-specific ODI score ranges 0 to $100 \%$, with higher scores indicating more disability related to back pain. The SF-36, a generic HRQoL questionnaire, consists of 36 self-administered questions which measure health status in eight domains of HRQoL: physical functioning (PF); role-physical (RP), or limitation in daily role functioning due to physical problems; role-emotional (RE), or limitation in daily role functioning due to emotional problems; bodily pain (BP); general health perception (GH); vitality (VT); social functioning (SF); and mental health perception (MH). The physical component summary (PCS) and mental component summary (MCS) scales summarize the eight SF-36 scale scores into two summary scores that give an overall assessment of HRQoL related to physical and mental health, respectively. The PCS includes four domains: PF, RP, BP, and GH. The MCS includes four domains: VT, $\mathrm{RE}, \mathrm{SF}$, and MH. It has been proved that the SF-36 PCS and MCS scales are valid, reliable, and equivalent for the Chinese adult population (20). Domain scores range from 0 to 100 , with higher scores indicating better health status (21).

\section{Adverse events (AEs)}

An $\mathrm{AE}$ was defined as any untoward medical occurrence in a patient or clinical investigation subject administered a pharmaceutical product and which does not necessarily have to have a causal relationship with this treatment (22). AEs were recorded at each study visit and were given instructions for the assignment of $\mathrm{AE}$ severity as mild, moderate or severe. Mild was defined as no change in physical activity with occasional medication use for relief of symptoms. Moderate included mild disruptions in daily physical activities and regular medication use for the alleviation of symptoms. Criteria for severe were major disruptions in normal daily activities, additional medication use and additional treatment above and beyond normal that may have included hospitalization. Treatment would be discontinued if serious AEs occurred. All of them completed the observation period and no patients withdrew from the trial for this reason.

\section{Statistical analysis}

Categorical variables were expressed as number or percentages and compared using the Chi-square or Fisher exact test. The Student $t$-test was used to compare the characteristics between two treatment groups. Means and standard deviations (SDs) were used to summarize those characteristics. A two-way repeated measures ANOVA was performed to compare the changes between treatment groups during the follow-up period throughout the treatment. All these statistical analyses were performed using SPSS 20.0, with 0.05 regarded as significant.

\section{Results}

A total of 112 subjects were enrolled ( $\mathrm{n}=38$ in Teriparatide group and $n=74$ in control group) and screened for 1 year in the study. Baseline demographic data and clinical characteristics including age, BMI, the results of metabolic, and bone absorptiometry tests showed no difference between the groups (Table 1). The results of metabolic and bone absorptiometry tests were obtained only once and showed the loss of bone mass and abnormal bone metabolism status at the start of the study. The overall clinical outcomes for the two groups at each time point were listed in Figure 1 and Tables 2,3. There was no significant difference between groups regarding the results of VAS, ODI, SF-36 PCS, and MCS scores at baseline.

The VAS scores showed a trend towards improvement and decreased continuously during the whole treatment course (Tables 2,3) in both groups. The VAS scores in Teriparatide group were significantly lower $(\mathrm{P}<0.05)$ than in control group at all time points. At the end of study, the mean VAS scores had declined by $84.7 \%$ (6.84) from baseline in Teriparatide group and by $35.4 \%$ (2.68) in control group $(\mathrm{P}<0.05)$. In other words, Teriparatide showed a more significant effect on back pain relief $(\mathrm{P}<0.05)$.

Changes in ODI scores are shown in Table 2 and Figure 1. This value declined in both Teriparatide group and control group. But the ODI scores in Teriparatide group were significantly lower at the end of study, and Table 3 shows the scores for overall ODI reduced by 62.62 from baseline in the Teriparatide group compared with 29.32 in control 
Table 1 Background demographic data and baseline characteristics of patients in two groups

\begin{tabular}{|c|c|c|c|}
\hline Characteristics of patients & Teriparatide group $(n=38)$ & Control group $(n=74)$ & $P$ \\
\hline Age (years) & $70.24 \pm 8.49$ & $71.89 \pm 6.87$ & 0.269 \\
\hline Sex (female) & $38(100.0)$ & $74(100.0)$ & - \\
\hline $\mathrm{BMI}\left(\mathrm{kg} / \mathrm{m}^{2}\right)$ & $22.26 \pm 3.31$ & $23.26 \pm 3.68$ & 0.16 \\
\hline Bone mineral density at lumbar spine (SD) & $-2.15 \pm 1.72$ & $-2.22 \pm 1.90$ & 0.84 \\
\hline Serum phosphorus (mmol/L) & $1.06 \pm 0.18$ & $1.10 \pm 0.22$ & 0.42 \\
\hline Serum calcium (mmol/L) & $2.27 \pm 0.16$ & $2.26 \pm 0.20$ & 0.42 \\
\hline Serum alkaline phosphatase (U/L) & $71.39 \pm 25.28$ & $100.95 \pm 253.27$ & 0.323 \\
\hline Number of OVCFs & $38(100.0)$ & $74(100.0)$ & - \\
\hline Initial SF-36 PCS & $22.45 \pm 10.15$ & $25.93 \pm 14.28$ & 0.141 \\
\hline Initial SF-36 MCS & $36.14 \pm 16.91$ & $41.18 \pm 17.46$ & 0.145 \\
\hline
\end{tabular}

Data presented as mean \pm SD and values are numbers (percentages) unless stated otherwise. BMI, body-mass index; SD, standard deviation; OVCFs, osteoporotic vertebral compression fractures; VAS, visual analogue score; ODI, Oswestry disability index; SF-36, short form 36 questionnaire; PCS, physical component summary; MCS, mental component summary
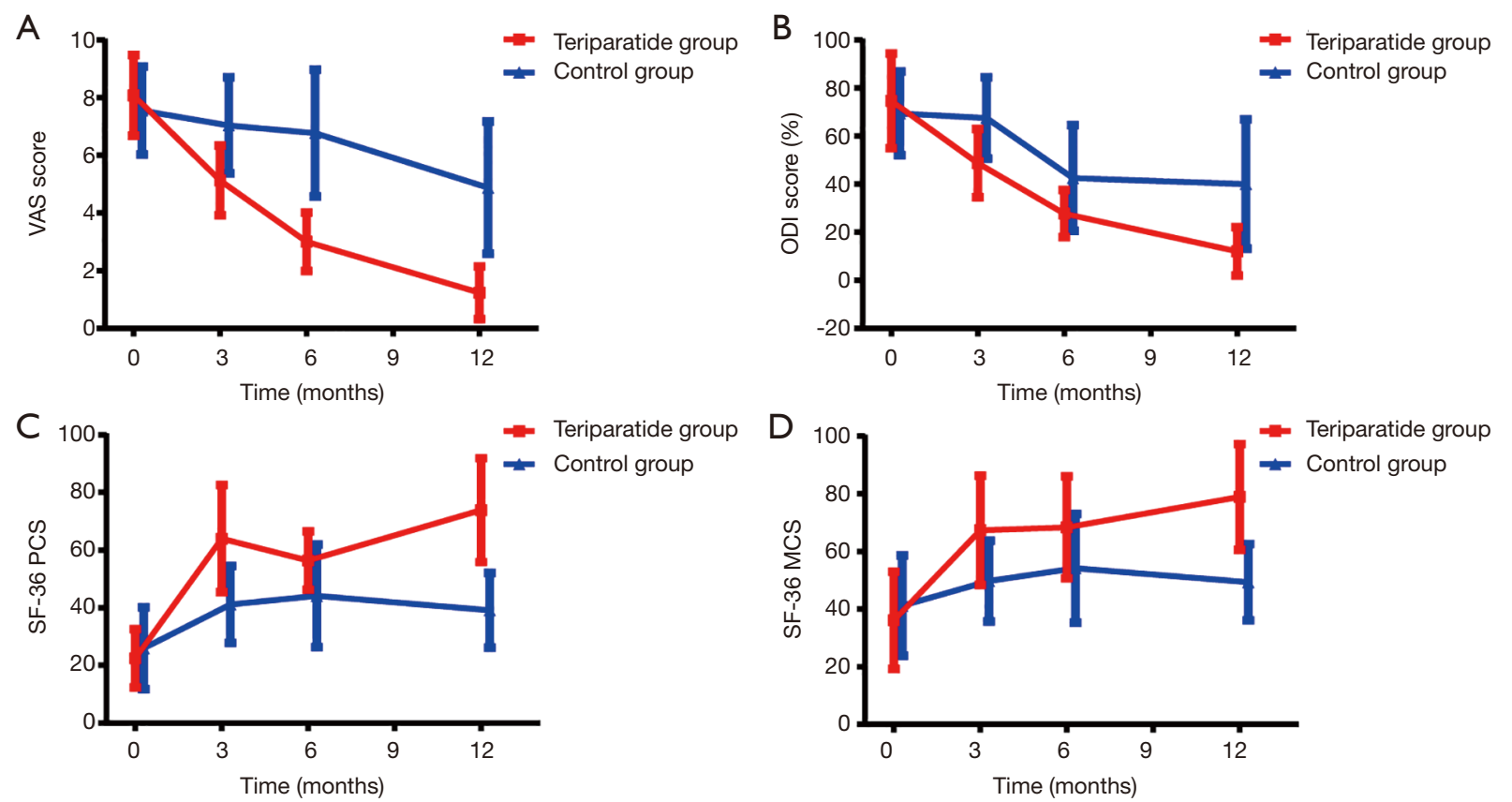

Figure 1 VAS (A), ODI scores (B) and SF-36 PCS index (C), SF-36 MCS index (D) for recruited patients in Teriparatide and control group during follow-up. Bars show standard error. VAS, visual analogue score; ODI, Oswestry disability index; SF-36, short form 36 questionnaire; PCS, physical component summary; MCS, mental component summary. 
Table 2 Overall clinical outcomes for two groups at each time point

\begin{tabular}{|c|c|c|c|c|c|c|c|c|}
\hline \multirow{3}{*}{ Variables } & \multicolumn{8}{|c|}{ Months after initiation } \\
\hline & \multicolumn{4}{|c|}{ Teriparatide group $(n=38)$} & \multicolumn{4}{|c|}{ Control group $(n=74)$} \\
\hline & Baseline & 3 months & 6 months & 12 months & Baseline & 3 months & 6 months & 12 months \\
\hline VAS & $8.08 \pm 1.40$ & $5.13 \pm 1.21^{*}$ & $3.00 \pm 1.01^{*}$ & $1.24 \pm 0.91^{*}$ & $7.55 \pm 1.53$ & $7.04 \pm 1.67$ & $6.77 \pm 2.20^{*}$ & $4.88 \pm 2.29^{*}$ \\
\hline ODI (\%) & $74.66 \pm 19.73$ & $48.71 \pm 14.26^{*}$ & $27.71 \pm 9.83^{*}$ & $12.05 \pm 10.05^{\star}$ & $69.41 \pm 17.47$ & $67.54 \pm 16.86$ & $42.54 \pm 22.06^{*}$ & $40.10 \pm 26.99^{*}$ \\
\hline SF-36 MCS & $36.14 \pm 16.91$ & $67.35 \pm 19.06^{*}$ & $68.39 \pm 17.69^{*}$ & $78.94 \pm 18.39^{*}$ & $41.18 \pm 17.46$ & $49.77 \pm 14.04^{*}$ & $54.20 \pm 18.81^{*}$ & $49.39 \pm 13.30^{*}$ \\
\hline
\end{tabular}

Data presented as mean \pm SD unless stated otherwise. * indicates $\mathrm{P}<0.05$ compared with value at baseline of their own groups. VAS, visual analogue score; ODI, Oswestry disability index; SF-36, short form 36 questionnaire; PCS, physical component summary; MCS, mental component summary; SD, standard deviation.

Table 3 Differences value between two groups

\begin{tabular}{lcccc}
\hline D value & Teriparatide group $(\mathrm{n}=38)$ & Control group $(\mathrm{n}=74)$ & $\mathrm{t}$ & $\mathrm{P}$ \\
\hline VAS & $-6.84 \pm 1.78$ & $-2.68 \pm 2.24$ & 6.72 & $<0.05$ \\
ODI & $-62.62 \pm 22.61$ & $-29.32 \pm 29.69$ & -9.061 & $<0.05$ \\
PCS & $51.39 \pm 22.46$ & $13.19 \pm 18.12$ & -7.396 & $<0.05$ \\
MCS & $42.80 \pm 25.37$ & $8.21 \pm 18.96$ & $<.05$ \\
\hline
\end{tabular}

Data presented as mean \pm SD unless stated otherwise. D value, difference value; VAS, visual analogue score; ODI, Oswestry disability index; SF-36, short form 36 questionnaire; PCS, physical component summary; MCS, mental component summary; SD, standard deviation.

groups, which was statistically significant.

At baseline, between-group differences were not statistically significant in neither PCS nor MCS $(\mathrm{P}>0.05$ for all). However, the SF-36 physical component and mental component followed the same trend during the treatment course, and showed significant improvement at the end of study (Table 2). Mean changes in both SF-36 PCS and MCS from baseline were positive, indicating better HRQoL at the end of the study. What's more, the changes from baseline to endpoint in Teriparatide group were relatively larger compared with that of control group $(\mathrm{P}<0.05)$, showing a notably superior response to Teriparatide than calcium plus vitamin D supplements regarding the improvement of HRQoL (Table 3).

AEs were systematically recorded at all patient visits. Overall, self-administered injections of Teriparatide and the oral calcium plus vitamin D supplements were well tolerated in spite of some cases of slight discomforts. Twelve (31.6\%) patients complained of slight nausea and dizziness in Teriparatide group. Discomfort generally resolved as the study progressed with no further consequences and did not result in any drop-out. By contrast, all patients in the control group were satisfied with therapy and no side effect was reported. In short, more AEs were documented in Teriparatide group $(\mathrm{P}<0.0001)$.

\section{Discussion}

In this retrospective observational study of postmenopausal women with OVCFs to report quality of life and back pain in routine clinical practice, treatment with Teriparatide resulted in significantly greater decrease in basal pain and immediate improvement in impaired quality of life, compared with calcium plus vitamin D supplements.

Musculoskeletal symptoms are relatively common in postmenopausal women with OVCFs, with back pain being the most frequent and the earliest. In postmenopausal women with osteoporosis, vertebral fracture itself is an important etiology of back pain, and the degree of back pain increases with aggravating vertebral fracture. Back pain seems to contribute more to disability by itself than vertebral fracture does (23). Several previous studies had 
demonstrated the use of Teriparatide can effectively reduce the incidence and severity of back pain in postmenopausal women with osteoporosis. Genant et al. previously reported that women randomized to PTH $(20 \mu \mathrm{g})$ had a $31 \%$ reduced relative risk of moderate or severe back pain and a $57 \%$ reduced risk of severe back pain (24). Two studies of Neer et al. and Body et al. had reported that fewer women treated with PTH suffered back pain when compared with those treated with placebo $(14,15)$. However, it was uncertain whether Teriparatide alleviated back pain of postmenopausal women with OVCFs. In the current study, we demonstrated the analgesic effect on basal and induced pain of OVCFs compared to calcium plus vitamin D supplements in postmenopausal women with OVCFs, consistent with those results above. What's more, we found that the mean reduction in the VAS score was approximately $84.65 \%$ in Teriparatide group, exceeding the reduction of $30 \%$ that is considered as clinically minimally important difference (MID) (25). While a much smaller reduction of $35.36 \%$ was observed in the control group, suggesting the superiority of Teriparatide over calcium plus vitamin D supplements.

To the best of our knowledge, this current study is the first to demonstrate an improvement in HRQoL with Teriparatide treatment. In the past few years, some researchers focused on the HRQoL of patients with OVCFs, and demonstrated that the OVCFs were closely associated with the reduced HRQoL $(26,27)$. In addition, they also put forward evidences that vertebral fractures can diminish physical function and emotional status; reduce capacity to perform activities of daily living, and overall decline in a patient's well-being $(6,28,29)$. In fact, the need to "to rethink vertebral osteoporosis and to focus more closely than bitherto on the quality of life of these patients" has been recognized in 1992 in an editorial (30). Improvements in HRQoL associated with treatment would undoubtedly be of particular relevance to patients. In this study, the HRQoL improved early in the therapy course, as early as 3 months, and remained elevated at each time point until the study completion. Significantly more improvement in the Teriparatide group achieved HRQoL gains compared to the control group, in terms of both physical and mental component. These data suggest that Teriparatide may be a more potent anti-osteoporosis drug in postmenopausal females with OVCFs.

In this relatively short course of treatment, serious AEs secondary to the medication were not reported in the two treatment groups, ensuring high compliance of patients to complete the course. In our study, no patients stopped the drugs due to the AEs, and all of 112 patients had finished the course.

The main drawback of our study include the relatively small number of subjects, which were groups two groups according to their choices on different treatments, but the numbers were sufficient to detect statistically significant differences in the outcomes of interest. Secondly, a relatively short observation window limits the extrapolation of our final results to the generalization of a 24-month course to certain extent. Finally, in blood samples, bone turnover markers were not included; however, the positive biochemical marker response after treatment of Teriparatide has been proved an anabolic biologic response in the bone $(31,32)$. What's more, routine blood tests of bone turnover markers were very difficult to achieve, especially in the orthopedics clinic or during the outpatient followup. Furthermore, the aim of this study was to focus mainly on the effect of Teriparatide on reducing back pain, and improving HRQoL for postmenopausal women with OVCFs, rather than to explore the mechanism of action in depth. That's why these bone turnover markers were not included in our study. To overcome these shortcomings, we set strict inclusion and exclusion criteria trying to ensure that improvements in HRQoL were due to treatment as much as possible. We believed that the improvements of pain and HRQoL could be proved by the effect of Teriparatide.

\section{Conclusions}

In postmenopausal women with OVCFs and the consequent persistent back pain and impaired HRQoL, treatment with Teriparatide was associated with more profound therapeutic effects and more AEs compared with calcium plus vitamin D supplements. A more extensive randomized controlled trial could allow the assessment of longer-term outcomes and provide additional information about potential AEs.

\section{Acknowledgments}

We thank all co-authors for support from data collection to manuscript revision.

Funding: This work was supported by the Natural Science Foundation of China (No. 31570976), and Science and Technology Program of Guangzhou, China (No. 201604020148). The content is solely the responsibility of the authors and does not necessarily represent the official views of the NSFC. 


\section{Footnote}

Reporting Checklist: The authors have completed the STROBE reporting checklist. Available at http://dx.doi. org/10.21037/apm-20-2333

Data Sharing Statement: Available at http://dx.doi. org/10.21037/apm-20-2333

Peer Review File: Available at http://dx.doi.org/10.21037/ apm-20-2333

Conflicts of Interest: All authors have completed the ICMJE uniform disclosure form (available at http://dx.doi. org/10.21037/apm-20-2333). The authors have no conflicts of interest to declare.

Ethical Statement: The authors are accountable for all aspects of the work in ensuring that questions related to the accuracy or integrity of any part of the work are appropriately investigated and resolved. The study was performed in compliance with the ethical principles outlined in the World Medical Association's Declaration of Helsinki (as revised in 2103) and was approved by the Institutional Review Board of the First Affiliated Hospital of Sun Yat-sen University (No. 2020-149) and individual consent for this retrospective analysis was waived.

Open Access Statement: This is an Open Access article distributed in accordance with the Creative Commons Attribution-NonCommercial-NoDerivs 4.0 International License (CC BY-NC-ND 4.0), which permits the noncommercial replication and distribution of the article with the strict proviso that no changes or edits are made and the original work is properly cited (including links to both the formal publication through the relevant DOI and the license). See: https://creativecommons.org/licenses/by-nc-nd/4.0/.

\section{References}

1. NIH Consensus Development Panel on Osteoporosis Prevention, Diagnosis, and Therapy. Osteoporosis prevention, diagnosis, and therapy. JAMA 2001;285:785-95.

2. Nevitt MC, Ettinger B, Black DM, et al. The association of radiographically detected vertebral fractures with back pain and function: a prospective study. Ann Intern Med 1998;128:793-800.

3. Melton LJ 3rd, Lane AW, Cooper C, et al. Prevalence and incidence of vertebral deformities. Osteoporos Int 1993;3:113-9.

4. Kanis JA, Geusens P, Christiansen C. Guidelines for clinical trials in osteoporosis. A position paper of the European Foundation for Osteoporosis and Bone Disease. Osteoporos Int 1991;1:182-8.

5. Greendale GA, Silverman SL, Hays RD, et al. Healthrelated quality of life in osteoporosis clinical trials. The Osteoporosis Quality of Life Study Group. Calcif Tissue Int 1993;53:75-7.

6. Ross PD, Ettinger B, Davis JW, et al. Evaluation of adverse health outcomes associated with vertebral fractures. Osteoporos Int 1991;1:134-40.

7. Oleksik A, Lips P, Dawson A, et al. Health-related quality of life in postmenopausal women with low BMD with or without prevalent vertebral fractures. J Bone Miner Res 2000;15:1384-92.

8. Barker KL, Javaid MK, Newman M, et al. Physiotherapy Rehabilitation for Osteoporotic Vertebral Fracture (PROVE): study protocol for a randomised controlled trial. Trials 2014;15:22.

9. Bilotta C, Bowling A, Nicolini P, et al. Older People's Quality of Life (OPQOL) scores and adverse health outcomes at a one-year follow-up. A prospective cohort study on older outpatients living in the community in Italy. Health Qual Life Outcomes 2011;9:72.

10. Gehlbach SH, Burge RT, Puleo E, et al. Hospital care of osteoporosis-related vertebral fractures. Osteoporos Int 2003;14:53-60.

11. Finnern HW, Sykes DP. The hospital cost of vertebral fractures in the EU: estimates using national datasets. Osteoporos Int 2003;14:429-36.

12. Cauley JA. Public health impact of osteoporosis. J Gerontol A Biol Sci Med Sci 2013;68:1243-51.

13. Cosman F. Parathyroid hormone treatment for osteoporosis. Curr Opin Endocrinol Diabetes Obes 2008; 15:495-501.

14. Neer RM, Arnaud CD, Zanchetta JR, et al. Effect of parathyroid hormone (1-34) on fractures and bone mineral density in postmenopausal women with osteoporosis. $\mathrm{N}$ Engl J Med 2001;344:1434-41.

15. Body JJ, Gaich GA, Scheele WH, et al. A randomized double-blind trial to compare the efficacy of teriparatide [recombinant human parathyroid hormone (1-34)] with alendronate in postmenopausal women with osteoporosis. J Clin Endocrinol Metab 2002;87:4528-35.

16. Gallagher JC, Genant HK, Crans GG, et al. Teriparatide reduces the fracture risk associated with increasing number 
and severity of osteoporotic fractures. J Clin Endocrinol Metab 2005;90:1583-7.

17. Nakajima A, Shimoji N, Shiomi K, et al. Mechanisms for the enhancement of fracture healing in rats treated with intermittent low-dose human parathyroid hormone (1-34). J Bone Miner Res 2002;17:2038-47.

18. Hodsman AB, Bauer DC, Dempster DW, et al. Parathyroid hormone and teriparatide for the treatment of osteoporosis: a review of the evidence and suggested guidelines for its use. Endocr Rev 2005;26:688-703.

19. Sriwatanakul K, Kelvie W, Lasagna L, et al. Studies with different types of visual analog scales for measurement of pain. Clin Pharmacol Ther 1983;34:234-9.

20. Lam CL, Tse EY, Gandek B, et al. The SF-36 summary scales were valid, reliable, and equivalent in a Chinese population. J Clin Epidemiol 2005;58:815-22.

21. Adorno ML, Brasil-Neto JP. Assessment of the quality of life through the SF-36 questionnaire in patients with chronic nonspecific low back pain. Acta Ortop Bras 2013;21:202-7.

22. James EC, Dunn D, Cook AD, et al. Overlap between adverse events (AEs) and serious adverse events (SAEs): a case study of a phase III cancer clinical trial. Trials 2020;21:802.

23. Edmond SL, Kiel DP, Samelson EJ, et al. Vertebral deformity, back symptoms, and functional limitations among older women: the Framingham Study. Osteoporos Int 2005; 16:1086-95.

24. Genant HK, Halse J, Briney WG, et al. The effects of teriparatide on the incidence of back pain in

Cite this article as: Chen Z, Lin W, Zhao S, Mo X, Yuan W, Cheung WH, Fu D, Chen B. Effect of Teriparatide on pain relief, and quality of life in postmenopausal females with osteoporotic vertebral compression fractures, a retrospective cohort study. Ann Palliat Med 2021;10(4):4000-4007. doi: 10.21037/ apm-20-2333 postmenopausal women with osteoporosis. Curr Med Res Opin 2005;21:1027-34.

25. Dworkin RH, Turk DC, Wyrwich KW, et al. Interpreting the clinical importance of treatment outcomes in chronic pain clinical trials: IMMPACT recommendations. J Pain 2008;9:105-21.

26. Tosteson AN, Gabriel SE, Grove MR, et al. Impact of hip and vertebral fractures on quality-adjusted life years. Osteoporos Int 2001;12:1042-9.

27. Silverman SL, Minshall ME, Shen W, et al. The relationship of health-related quality of life to prevalent and incident vertebral fractures in postmenopausal women with osteoporosis: results from the Multiple Outcomes of Raloxifene Evaluation Study. Arthritis Rheum 2001;44:2611-9.

28. Burger H, Van Daele PL, Grashuis K, et al. Vertebral deformities and functional impairment in men and women. J Bone Miner Res 1997;12:152-7.

29. Gold DT, Smith SD, Bales CW, et al. Osteoporosis in late life: does health locus of control affect psychosocial adaptation? J Am Geriatr Soc 1991;39:670-5.

30. Kanis JA, Minne WH, Meunier PJ, et al. Quality of life and vertebral osteoporosis. Osteoporos Int 1992;2:161-3.

31. McClung MR, San Martin J, Miller PD, et al. Opposite bone remodeling effects of teriparatide and alendronate in increasing bone mass. Arch Intern Med 2005;165:1762-8.

32. Glover SJ, Eastell R, McCloskey EV, et al. Rapid and robust response of biochemical markers of bone formation to teriparatide therapy. Bone 2009;45:1053-8. 